biological significance of circulating antibodies to dietary antigens. Ahlstedt's review of these subjects is impressive and authoritative. There is a ample consideration of host defence to parasitic and other gut infections; it has, for example, long been a conundrum that IgE and eosinophils are hall-marks of allergic reactions yet are clearly part of the reaction to such infections. Mayrhofer and Beeson each lay the foundations for a detailed consideration of the control of immune reactions that on the one hand limit the extent of microbial invasion, and on the other can damage the gut. Thus it is felicitous that the book includes Rosen's description of gut disorders in immunodeficiency and Seligman's account of alpha-chain disease as these disorders provide vital clues to the regulatory processes whose breakdown initiates allergic disease. There is an impressive attempt to provide a logical continuity between basic immunology and the immunology of common diseases such as coeliac disease and ulcerative colitis. These sections are most successful when the contributors have attempted a slow but purposive progression from physiological analysis to disease phenomena. A good example is Anne Ferguson's description of how simple animal models can be adapted for research on coeliac disease. In contrast those sections based on uninterpretable clinical observations are less successful, especially when clouded by speculation that transcends the meagre data available. It is sobering to reflect that Dicke's simple observations, made in 1950, about the relation between coeliac disease and gluten has given us $90 \%$ of the total useful knowledge concerning the pathogenesis of the disorder derived in three decades of intense effort.

This is a splendid book for the clinician, scientist or microbiologist who wants to appreciate the direction of modern thought about gut immunology and immunopathology. For a change, the lengthy discussion sections help in this regard. Indeed, it would be a positive advantage not to have read most earlier tracts on the subject. It is not a textbook and is certainly too stimulating and refreshing to have any value for examination candidates. The only prerequisites are an unbiased mind and familiarity with a simple introductory text on immunology.

A. M. Denman

\title{
The specificity and action of animal, bacterial and plant toxins
}

Edited by P. Cuatrecasas. 1977. Andover, Hants.: Chapman and Hall Ltd. Pp. ix and 345. $£ 15 \cdot 00$.

This book summarises recent advances in knowledge of the nature and mechanism of action of a group of well characterised toxins. Cuatrecasas aims to focus the reader's attention not on the disease-causing properties of these toxins but on their potential value as probes of membrane structure and function and cell physiology. Most of the toxins dealt with are bacterial products (cholera toxin, diphtheria toxin, the lethal toxin of Pseudomonas aeruginosa, tetanus toxin, botulinum toxin, the cytolytic toxins and colicin E3). However, there is a chapter on the plant toxins abrin and ricin, and two animal toxins, $\beta$-bungarotoxin and batrachotoxin, are also included. Each contribution is a detailed critical review prepared by an expert in the field.

One of the main themes is that several toxins, especially cholera toxin, diphtheria toxin, colicin E3 and the plant toxins abrin and ricin (from the seeds of the legume Abrus precatorius and the castor bean plant Ricinus communis, respectively, exhibit common features. They bind to specific receptors on the surfaces of susceptible cells and, after a lag period during which toxin-receptor complexes probably move in the lateral plane of the membrane, penetrate the membrane to act on their specific target sites. Most of these toxins are also similar in that their molecules consist of separable regions, a binding site and an active (toxic) site. The usefulness of radiolabelled toxin with high specific activity to study binding is emphasised, as are the problems of identifying specific receptors. The receptor for diphtheria toxin for instance has not yet been identified because there are few binding sites per cell. The least well-understood aspect of the action of these toxins is clearly the mechanism of penetration through the cell membrane. 
The molecular basis of toxin action in each case is thoroughly and clearly described. Among the most interesting accounts are the analysis by Bennet and Cuatrecasas of the kinetics of activation of adenylate cyclase by cholera toxin, the description by Collins of the similarities between diphtheria toxin and the lethal toxin of Pseudomonas aeruginosa, both of which cause covalent attachment of ADP ribose to elongation factor 2, and Holland's account of the hydrolysis of $16 \mathrm{~S}$ RNA in 70S ribosomes by colicin $\mathrm{E}_{3}$.

The summaries relating to tetanus toxin (by Bizzini) and botulinum toxin (by Simpson) are both timely as these toxins have not been reviewed recently. Bizzini outlines the current evidence that tetanus toxin blocks the presynaptic release of the inhibitory neurotransmitters glycine and $\gamma$-amino butyric acid in the CNS and argues that tetanus toxin is a useful pharmacological tool for studying the mechanism of secretion of neurotransmitter substances. His assessment of the contribution of Kryzhanovsky and co-workers is particularly useful to the general reader who might not have covered the Russian literature. Simpson's account of the mode of action of botulinum toxin and $\beta$-bungarotoxin from the venom of Bungarus multicinitus, both of which block acetyl choline release at peripheral nerve endings, serves to emphasise the value of these agents in studying trophic effects of nerve and muscle. The redistribution of acetyl-choline receptor sites after nerve section or toxin action would be such an application.

Present knowledge of the mode of action of the cytolytic (membrane-damaging) toxins is reviewed by Alouf. His account of the methods of studying membrane damage by observing the release of intracellular markers is of value to workers in this field. These toxins act directly on membrane components and induced permeability defects in susceptible cells. In some instances the target site in the membrane has been identified and Staphylococcus aureus $\beta$-toxin (sphingomyelinase C), Clostridium welchii $\alpha$-toxin (phospholipase C) and the cholesterol-specific, oxygen-labile toxins have been used as specific probes of membrane structure and function.

Finally, a detailed review of batrachotoxin is given by Albuquerque and Daly; this steroidal alkaloid toxin from a small neotropical frog depolarises excitable membranes by its ability to activate sodium channels selectively.

It must be emphasised that the contributions to this volume are highly specialised and the general reader might find some of the material difficult to digest. However, toxin action is a complex subject that derives its stimulus from many disciplines and if toxins are to gain general acceptance as selective inhibitors it is necessary to provide the potential user with as much relevant background information as possible and to indicate the limitations of our knowledge. This volume will undoubtedly be read with interest by "toxinologists" but it should also prove a useful source of information for other biologists. It is unfortunate that the index was not prepared with greater awareness of the needs of the non-specialised reader.

\section{J. P. ARbuthnotT}

\section{Antibiotics in general practice}

By Hillas Smith. 3rd ed., 1977. Tunbridge Wells, Kent: Pitman Medical Publishing Co. Ltd. Pp. $x$ and 413. $£ 8 \cdot 00$.

This book consists of two sections. The first, after general introductory chapters, treats each antimicrobial or group of antimicrobials in turn while the second deals with the management of individual infections. Here the author does not confine himself to antimicrobial aspects of therapy, but ranges widely over the many diagnostic and therapeutic problems that these infections present. The last few chapters deal with opportunistic infections, antibiotics in special circumstances (renal failure, the neonate and liver problems) and with chemoprophylaxis, while an earlier general chapter gives a sound practical approach to the problem of chemotherapy in undiagnosed fever. Other valuable features include tables of incompatibilities, a summary of dose schemes and adverse effects and, within the main text, many new summary tables. For example, because the need to desensitise patients to antituberculous drugs is rare, it is hard to know how best to do so, and suitable schemes are here provided. 